\title{
Simple 60-GHz MB-OFDM Ultrawideband RoF System Based on Remote Heterodyning
}

\author{
Oluyemi Omomukuyo, Student Member, IEEE, Manoj P. Thakur, Member, IEEE, and \\ John E. Mitchell, Member, IEEE
}

\begin{abstract}
A simple 60-GHz radio-over-fiber system employing a combination of uncorrelated optical heterodyning and envelope detection is proposed and experimentally demonstrated. At the central office, a multiband orthogonal f requency division multiplexing ultrawideband (MB-OFDM UWB) signal is used for the external modulation of a dual-arm Mach-Zehnder Modulator to generate an optical single-sideband signal, which is then coupled with an unmodulated free-running continuous wave laser. Optical heterodyne mixing at the base station and envelope detection at the customer unit are utilized. The experimental results confirm that photonic millimeter-wave signal generation and detection can be achieved without the need for complex optical phaselocked loops and high-frequency microwave sources. As a proof of concept, successful transmission of a $3.84 \mathrm{~Gb} / \mathrm{s}$ 16-QAM MB-OFDM UWB signal over 48-km standard single-mode fiber without chromatic dispersion compensation and a further 4-m wireless channel is experimentally demonstrated.
\end{abstract}

Index Terms-Microwave photonics, optical fiber communication, optical heterodyning, orthogonal frequency-division multiplexing (OFDM), radio-over-fiber (RoF), ultrawideband (UWB) communication.

\section{INTRODUCTION}

A $\mathrm{N}$ ultra-wideband (UWB) signal is defined as any radio signal with a $20 \%$ fractional bandwidth or a $10-\mathrm{dB}$ bandwidth of at least $500 \mathrm{MHz}$ [1]. UWB, a mature technology with efficient software and single-chip solutions available, exhibits advantages including high data rate, low power consumption and low cost. The $60-\mathrm{GHz}$ millimeter-wave (mm-wave) frequency band, where several gigahertz of license-free bandwidth are available, has recently been considered for the radio-over-fiber (RoF) delivery of multi-Gb/s UWB signals to end-users for bandwidth-intensive applications including high-definition television (HDTV), video-ondemand, wireless high-speed file transfer and wireless audio transmission [2].

Given that there are higher propagation losses in the mmwave frequency band than at the lower frequency bands, the cell-sizes of base-stations are relatively small, implying more base-stations will be needed to cover a given geographical

Manuscript received October 23, 2012; revised November 30, 2012; accepted December 13, 2012. Date of publication December 20, 2012; date of current version January 18, 2013.

The authors are with the Department of Electronic and Electrical Engineering, University College London, London WC1E 7JE, U.K (e-mail: o.omomukuyo@ee.ucl.ac.uk; m.thakur@ee.ucl.ac.uk; j.mitchell@ ee.ucl.ac.uk).

Color versions of one or more of the figures in this letter are available online at http://ieeexplore.ieee.org. area. It is therefore crucial, from an economic point of view, to simplify the optical millimeter-wave generation, delivery and data recovery. Consequently, numerous schemes have been proposed for optical millimeter-wave generation and optical frequency up-conversion. Some commonly used schemes include frequency/phase-locked correlated optical heterodyning [3] and the use of high-bandwidth external modulators alone, or in cascade with either another low-bandwidth external modulator or a directly modulated laser [2], [4], [5]. However, these schemes are disadvantaged by the need for signal control techniques or high-frequency electronic and electro-optic devices.

In this letter, we report and experimentally demonstrate for the first time to our knowledge, a $60-\mathrm{GHz}$ multi-band Orthogonal Frequency Division Multiplexing (MB-OFDM) UWB RoF system based on the uncorrelated optical heterodyne mixing of an optical single sideband (OSSB) MB-OFDM UWB signal with a free-running continuous-wave $(\mathrm{CW})$ laser in a broadband photodiode. The frequency offset between the two uncorrelated optical sources that beat at the photodiode is $60 \mathrm{GHz}$. Both optical sources are tunable lasers that utilize thermo-electric cooler (TEC) modules to ensure wavelength stability and accuracy throughout their tuning ranges. Using such lasers guarantees that the beat term falls within the $60-\mathrm{GHz}$ band.

It is well-known that the phase noise of the carrier generated by the beating of two uncorrelated optical sources on a photodetector significantly degrades the detection performance of the transmission system. This phase noise may manifest itself in the broadening of the spectral linewidth. In the absence of phase noise, the photodetected electrical signal at an intermediate frequency (IF) can be detected by extracting a carrier reference from it, followed by coherent demodulation. However, in the presence of phase noise, a carrier-recovery circuit might have difficulties in tracking rapid phase variations, resulting in degradation of the detection performance. Alternatively, these phase-noise effects can be avoided at baseband by utilizing a self-homodyning receiver [6] or an envelope detector with sufficient IF bandwidth [7]. The proposed system utilizes square-law envelope detection at the customer unit to down-convert the mm-wave wireless signal straight to baseband. Envelope detection is preferred over self-homodyning because it is cheaper and has been shown to provide better sensitivity [8].

Consequently, the proposed scheme significantly simplifies the optical mm-wave generation and data recovery as it 
doesn't require any high-bandwidth electro-optical device at the central office or high-frequency Local Oscillators (LOs) at either the central office or the customer unit; or optical phase-locking techniques to generate the $\mathrm{mm}$-wave wireless signal. This means that millimeter-wave independence is achieved, provided the frequency separation between the two optical sources is within the bandwidth of the broadband photodiode.

We experimentally demonstrate, using the proposed system, the successful transmission of a $3.84 \mathrm{~Gb} / \mathrm{s}$ 16-quadrature amplitude modulation (QAM) MB-OFDM UWB signal in the $60-\mathrm{GHz}$ band over up to $48-\mathrm{km}$ standard single-mode fiber (SSMF) and a further 4-m wireless channel.

\section{System Model}

Fig. 1 shows the block diagram of the proposed remote heterodyne system with envelope detection. One laser (signal laser) is used as the optical source to the Mach-Zehnder modulator (MZM) which is modulated by the MB-OFDM UWB signal. The phase fluctuations of the signal laser $\emptyset_{1}(t)$, as characterized by the Wiener-Lévy process, has zero mean and a variance of $2 \pi \beta|t|$, where $\beta$ is the two-sided $3-\mathrm{dB}$ laser linewidth of Lorentzian power density spectrum [9]. To introduce this phase fluctuation, the optical field at the output of the MZM is multiplied by the noisy carrier $e^{j \emptyset_{1}(t)}$, which is the laser phase noise. Consequently, the field of the optical UWB signal at the output of the MZM in the presence of the signal laser phase noise can be written as:

$$
\begin{aligned}
E_{1 p}(t)= & e^{j\left(2 \pi f_{L 1} t+\emptyset_{1}(t)\right)} \\
& +e^{j\left(2 \pi\left[f_{L 1}+f_{I F}\right] t+\emptyset_{1}(t)\right)} \cdot \sum_{k=\frac{-N}{2}+1}^{\frac{N}{2}} C_{k} e^{j 2 \pi f_{k} t}
\end{aligned}
$$

where $f_{L 1}, f_{I F}, N$ and $f_{k}$ are the center frequency of the signal laser, the carrier frequency of the UWB signal, the number of OFDM subcarriers and the frequency of the $k$ th subcarrier.

In a similar vein, the optical field at the output of the second laser (LO laser), in the presence of the LO laser phase noise, $e^{j \emptyset_{2}(t)}$ can be expressed as:

$$
E_{2 p}(t)=e^{j\left(2 \pi f_{L 2} t+\emptyset_{2}(t)\right)}
$$

where $f_{L 2}$ is the center frequency of the LO laser. The two optical signals are combined in the optical coupler resulting in the optical signal spectra shown in inset (a) of Fig. 1.

For simplicity, we have neglected both the fiber and wireless channels in this analysis. The generated photocurrent, $I_{1}(t)$, at the output of the square-law photodetector is:

$$
I_{1}(t)=\mathcal{R}\left\{\left(E_{1 p}(t)+E_{2 p}(t)\right) \cdot\left(E_{1 p}(t)+E_{2 p}(t)\right)^{*}\right\}
$$

where $\mathcal{R}$ is the responsivity of the photodetector and the superscript "*" carries out the complex conjugation operation. After some algebraic manipulations, it is easy to show that (3)

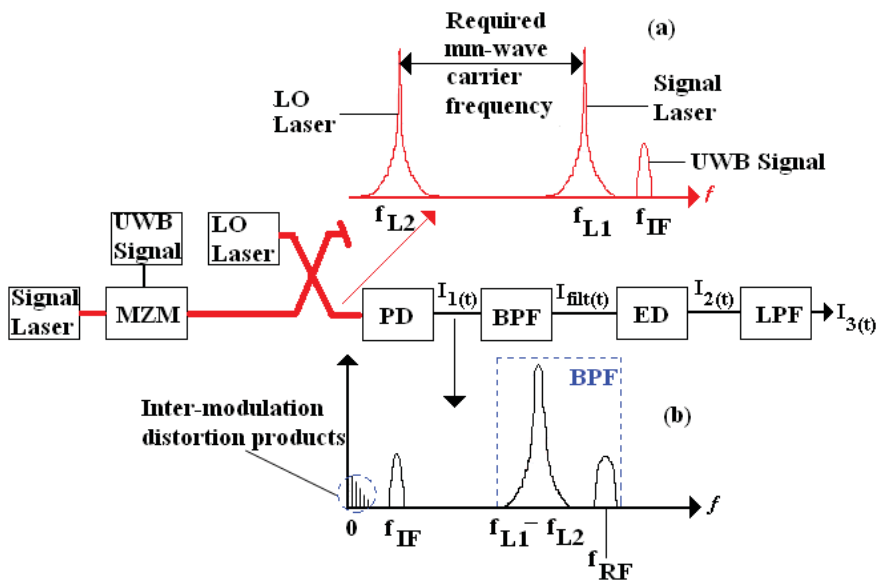

Fig. 1. Block diagram of the proposed system. Inset (a) Combined optical spectra. Inset (b) Photodetected signal spectra. MZM: Mach-Zehnder modulator. LO: local oscillator. PD: photodetector. BPF: band-pass filter. ED: envelope detector. LPF: low-pass filter.

can be expressed as:

$$
\begin{aligned}
I_{1}(t)=\mathcal{R} & \left\{2+\left[\left(\sum_{k=\frac{-N}{2}+1}^{\frac{N}{2}} C_{k} \cos \left(2 \pi f_{k} t\right)\right)^{2}\right.\right. \\
& \left.+\left[\sum_{k=\frac{-N}{2}+1}^{\frac{N}{2}} C_{k} \sin \left(2 \pi f_{k} t\right)\right)^{2}\right] \\
& +\left[2 \sum_{k=\frac{-N}{2}+1}^{\frac{N}{2}} C_{k} \cos \left(2 \pi\left[f_{I F}+f_{k}\right] t\right)\right] \\
& +\left[2 \cos \left(\epsilon_{1}(t)\right)\right] \\
& \left.+\left[2 \sum_{k=\frac{-N}{2}+1}^{\frac{N}{2}} C_{k} \cos \left(\epsilon_{2}+2 \pi f_{k} t\right)\right]\right\}
\end{aligned}
$$

where

$$
\begin{aligned}
& \epsilon_{1}(t)=2 \pi\left[f_{L 1}-f_{L 2}\right] t+\left(\emptyset_{1}(t)-\emptyset_{2}(t)\right) \\
& \epsilon_{2}(t)=2 \pi f_{R F} t+\left(\emptyset_{1}(t)-\emptyset_{2}(t)\right)
\end{aligned}
$$

and

$$
f_{R F}=f_{L 1}-f_{L 2}+f_{I F} .
$$

The first term in (4) is a D.C. component. The second term represents the second-order inter-modulation distortion products. The third term is a copy of the MB-OFDM UWB signal centred at a frequency of $f_{I F}$. The fourth term is a Radio Frequency (RF) carrier at a frequency of $\left(f_{L 1}-f_{L 2}\right)$, obtained from the beating of the two optical carriers with themselves. The final term is the desired MB-OFDM UWB signal at the RF frequency of $f_{R F}$. It is seen that the last two terms have random phase deviations of $\left(\phi_{1}(t)-\phi_{2}(t)\right)$. If $\phi_{1}(t)$ and $\phi_{2}(t)$ have spectral densities represented by $\eta_{1}$ and $\eta_{2} \mathrm{rps}^{2} / \mathrm{Hz}$ respectively, these last two terms will have spectral densities of $\left(\eta_{1}+\eta_{2}\right) \mathrm{rps}^{2} / \mathrm{Hz}$, resulting in a $3-\mathrm{dB}$ linewidth equal to the sum of the linewidths of the signal 


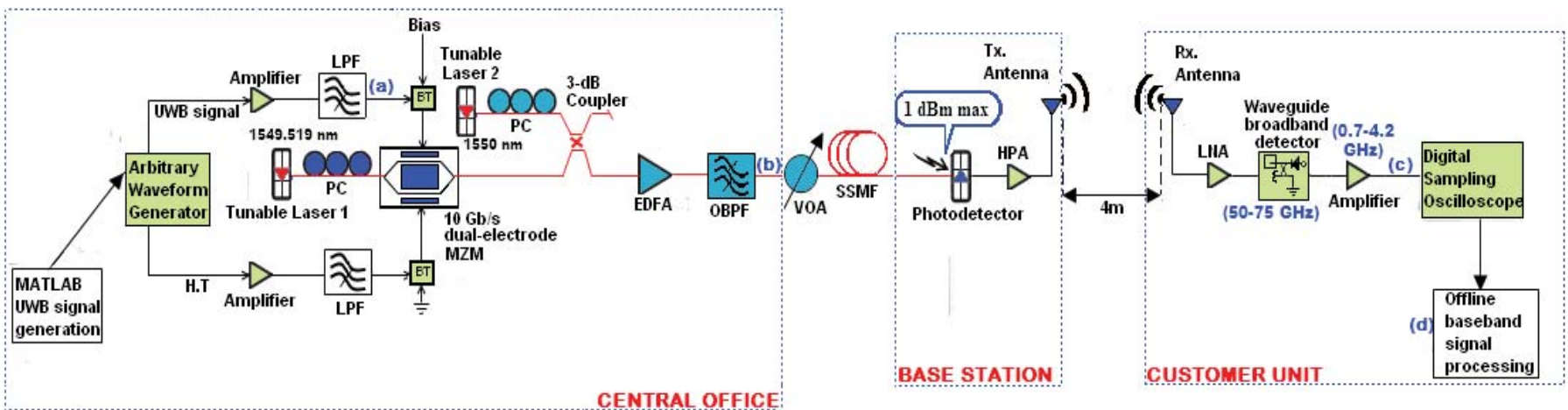

Fig. 2. Experimental setup of the proposed scheme. H.T: Hilbert transform. LPF: low-pass filter. BT: bias tee. PC: polarization controller. MZM: Mach-Zehnder modulator. EDFA: erbium-doped fiber amplifier. OBPF: optical band-pass filter. VOA: variable optical attenuator. SSMF: standard single-mode fiber. HPA: high-power amplifier. Tx.: transmit. Rx.: receive. LNA: low-noise amplifier.

and LO lasers [7]. All the terms in (4) are illustrated in the photodetected signal spectra shown in inset (b) of Fig. 1, with the increased linewidths of the last two terms clearly evident. The BPF shown in Fig. 1 removes the undesired components, leaving only the last two terms in (4). The filtered electrical signal, $I_{f i l t}(t)$ is down-converted by passing it through the envelope detector. Assuming an ideal square-law envelope detector, the down-converted signal $I_{2}(t)$ is written as:

$$
\begin{aligned}
I_{2}(t) & =\left|I_{\text {filt }}(t)\right|^{2} \\
& =4 \mathcal{R}^{2}\left\{\cos \left(\epsilon_{1}(t)\right)+\sum_{k=\frac{-N}{2}+1}^{\frac{N}{2}} C_{k} \cos \left(\epsilon_{2}+2 \pi f_{k} t\right)\right\}^{2} .
\end{aligned}
$$

After filtering $I_{2}(t)$ with the LPF, the higher-frequency components in (9) are eliminated, leaving the baseband signal, $I_{3}(t)$

$$
I_{3}(t)=4 \mathcal{R}^{2}\left\{\sum_{k=\frac{-N}{2}+1}^{\frac{N}{2}} C_{k} \cos \left(2 \pi\left[f_{I F}+f_{k}\right] t\right)\right\} .
$$

Equation (10) shows that no laser phase noise terms remain. This implies that the proposed system is, in principle, capable of avoiding the laser phase noise effects at baseband. In addition, the system is robust against chromatic dispersion because the desired UWB signal at the RF frequency is obtained by the beating of only two optical components, i.e. one optical carrier and the optical UWB sideband.

\section{EXPERIMENTAL SETUP}

The experimental setup of the proposed RoF system is shown in Fig. 2. The UWB signal is generated offline in MATLAB as a single-column ASCII text file and then loaded into a Tektronix AWG7122B Arbitrary Waveform Generator (AWG) operating at a sampling rate of $10.8 \mathrm{GS} / \mathrm{s}$. The modulating MB-OFDM UWB at point (a) in Fig. 2, spans from $1.5 \mathrm{GHz}$ to $3.084 \mathrm{GHz}$ and comprises three $528 \mathrm{MHz}$-wide sub-bands, each sub-band modulated using 16-QAM. The bit rate of each sub-band is $1.28 \mathrm{~Gb} / \mathrm{s}$, resulting in an aggregate bit rate of $3.84 \mathrm{~Gb} / \mathrm{s}$.

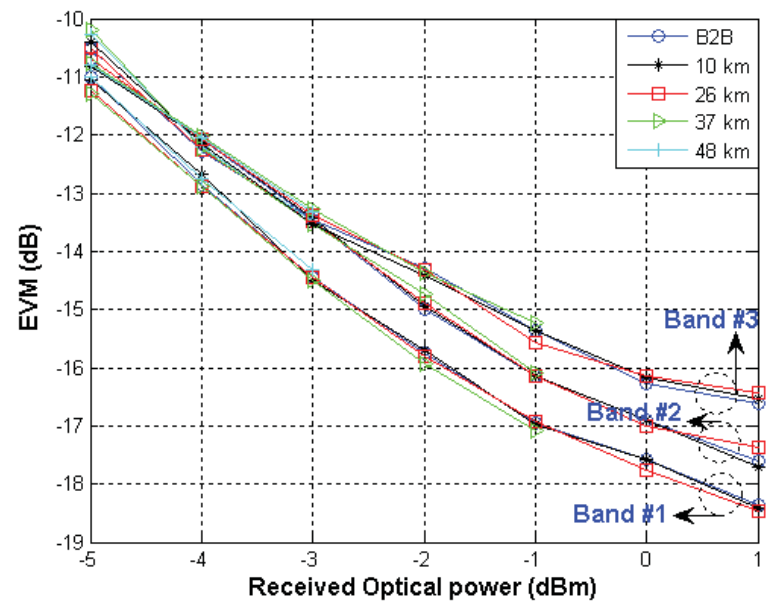

Fig. 3. EVM of the three UWB sub-bands as a function of received optical power for a fixed wireless distance of $2.4 \mathrm{~m}$ and different SSMF lengths.

To generate the optical data signal at the central office, the AWG is operated in dual-output mode. The first AWG output is the UWB signal while the second output is the Hilbert transform of the UWB signal. These two outputs are amplified by two 16-dB gain, RF amplifiers and then filtered by two 7.5-GHz, low-pass filters (LPFs) to remove the alias components produced from the digital-to-analog (DAC) conversion at the AWG. These filtered signals are then used as the electrical drives to a Fujitsu $10-\mathrm{Gb} / \mathrm{s}$ dual-electrode MZM $(\mathrm{V} \pi=3.1 \mathrm{~V})$ biased at its quadrature point. The optical source to the MZM is a tunable laser with linewidth of $800 \mathrm{kHz}$ and center emission wavelength of $1549.51 \mathrm{~nm}$. An OSSB is generated at the output of the MZM. This OSSB signal is combined in a 3-dB optical coupler with another $\mathrm{CW}$ signal emitted from another tunable laser with linewidth of $100 \mathrm{kHz}$ and center emission wavelength of $1550 \mathrm{~nm}$. The frequency difference between the two optical sources is chosen to yield the desired $60-\mathrm{GHz}$ $\mathrm{mm}$-wave carrier frequency. A polarization controller ensures maximum coupling of the two optical signals is achieved. The combined optical signals are then amplified by an Erbiumdoped fiber amplifier (EDFA) before being filtered using a 0.8-nm, tunable optical band-pass filter (OBPF) to suppress the out-of-band amplified spontaneous emission (ASE) noise. 


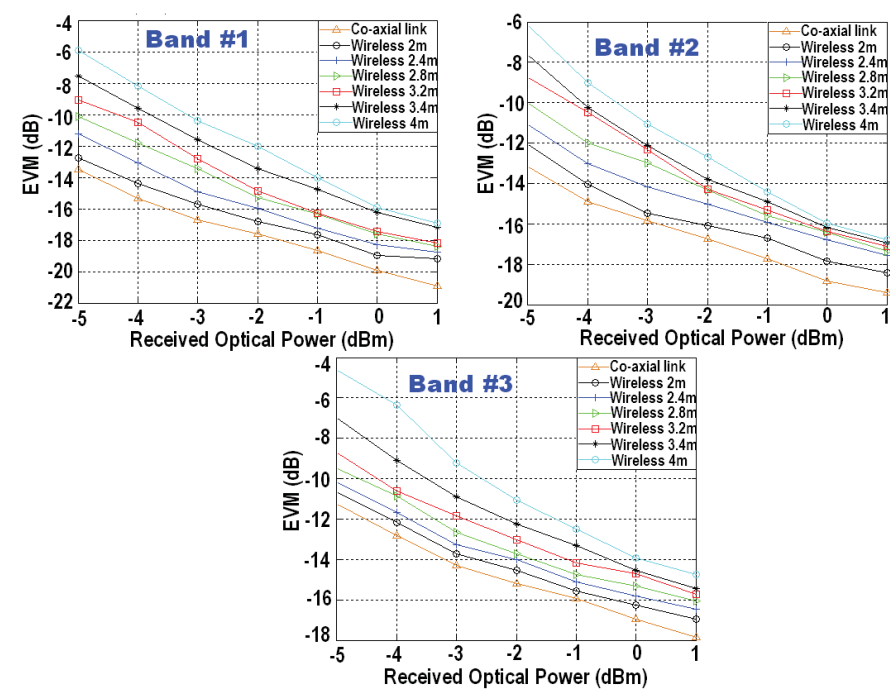

Fig. 4. EVM as a function of received optical power for a fixed SSMF length of $26 \mathrm{~km}$ and different wireless distances (direct co-axial link also included).

The optical signals are then transmitted through up to $48-\mathrm{km}$ standard single-mode fiber (SSMF).

A variable optical attenuator (VOA) is used to maintain a maximum of $1 \mathrm{dBm}$ input to a $70-\mathrm{GHz}$ broadband photodetector located at the base station. The attenuation of the VOA is varied to enable the Error Vector Magnitude (EVM) performance of the system to be analyzed as a function of the received optical power. After photodetection, the $60-\mathrm{GHz}$ UWB signal is amplified by a $30-\mathrm{dB}$ gain, high-power amplifier (HPA) before being applied to an antenna for up to $4 \mathrm{~m}$ wireless transmission. Both the antennas used in the experimental setup are dual-reflector Cassegrain antennas with frequency range of $50-75 \mathrm{GHz}, 30-\mathrm{dBi}$ gain and $3-\mathrm{dB}$ beam width of $3.1^{\circ}$. At the customer unit, the received wireless 60-GHz UWB signal is amplified by a $30-\mathrm{dB}$ gain, low-noise amplifier (LNA). Down-conversion of the mm-wave UWB signal is achieved by linearly detecting its envelope using a waveguide broadband detector with a frequency range of 50-75 GHz. The down-converted signal is further amplified by a $30-\mathrm{dB}$ gain amplifier with a frequency range of 0.7-4.2 GHz and then captured by a Tektronix DPO 72004, 20-GHz, real-time oscilloscope operated at a sampling rate of $50 \mathrm{GS} / \mathrm{s}$. Baseband digital signal processing including one-tap equalization, demodulation, demapping and EVM computation is performed offline in MATLAB.

\section{RESUlts AND Discussion}

The system performance is tested for a variation of the received optical power at a bit rate of $3.84 \mathrm{~Gb} / \mathrm{s}$. The peak-to-peak amplitudes of the UWB signals from the AWG are kept fixed at $0.8 \mathrm{~V}$. For a fixed wireless distance of $2.4 \mathrm{~m}$, the received optical power to the photodetector in the base station is varied by tuning the attenuation of the VOA. Fig. 3 shows the EVM variation of the three UWB sub-bands with the received optical power for five optical transmission cases: back-to-back, $10 \mathrm{~km}, 26 \mathrm{~km}, 37 \mathrm{~km}$ and $48 \mathrm{~km}$ of SSMF. The EVM is limited by electrical noise at low received optical power. Increasing the received optical power improves the EVM due to an increase in the received signal-to-noise ratio (SNR). For an optical receiver sensitivity of $0 \mathrm{dBm}$, an EVM below $-16 \mathrm{~dB}$ is obtained for all the three bands. Fig. 3 also shows that the system is robust against fiber chromatic dispersion, with hardly any power penalty obtained for the various fiber lengths used. Transmission over longer fiber spans is therefore possible but would be dependent on the available amplification power budget. In order to investigate the system performance dependence on the wireless distance, the fiber length is fixed at $26 \mathrm{~km}$ and with all other parameters constant, the wireless distance is varied from 2 to $4 \mathrm{~m}$. As can be seen in Fig. 4, the EVM performance degrades with longer wireless distances due to increase in the free-space path loss.

\section{Conclusion}

A 60-GHz RoF system which considerably simplifies optical mm-wave generation and wireless signal demodulation has been proposed and experimentally demonstrated. The system utilizes optical heterodyne mixing of an OSSB UWB signal with another free-running unmodulated laser in a photodiode and envelope detection to achieve reduced overall system complexity by avoiding any analog frequency/phase-locking mechanism or high-frequency electro-optic and RF devices. Using the proposed system, multiple wide-band 16-QAM OFDM channels have been successfully transmitted over 48-km SSMF without any chromatic dispersion compensation and a further 4-m wireless distance. The system also demonstrates impressive robustness to phase noise and fiber chromatic dispersion, the only limitation imposed in fiber distribution being the available amplification power budget.

\section{REFERENCES}

[1] Federal Communications Commission, "Revision of part 15 of the Commission's rules regarding ultra-wideband transmission systems," FCC, Washington, DC, Tech. Rep. FCC 02-48, Apr. 2002.

[2] M. Beltran, et al., "Performance of a 60-GHz DCM-OFDM and BPSKimpulse ultra-wideband system with radio-over-fiber and wireless transmission employing a directly-modulated VCSEL," IEEE J. Sel. Areas Commun., vol. 29, no. 6, pp. 1295-1303, Jun. 2011.

[3] J. P. Yao, "Microwave photonics," J. Lightw. Technol., vol. 27, no. 3, pp. 314-335, Feb. 1, 2009.

[4] G. H. Nguyen, B. Cabon, and Y. L. Guennec, "Generation of $60-\mathrm{GHz}$ MB-OFDM signal-over-fiber by up-conversion using cascaded external modulators," J. Lightw. Technol., vol. 27, no. 11, pp. 1496-1502, Jun. 1, 2009.

[5] O. Omomukuyo, M. P. Thakur, and J. E. Mitchell, "Experimental performance analysis of MB-OFDM ultra-wideband radio-overfiber signals in the $60-\mathrm{GHz}$ band using a commercially-available DFB laser," in Proc. ICTON 2012, Coventry, U.K., pp. 1-4, paper Mo.C3.4.

[6] A. H. M. R. Islam, et al., "Millimeter-wave radio-over-fiber system based on heterodyned unlocked light sources and self-homodyned RF receiver," IEEE Photon. Technol. Lett., vol. 23, no. 8, pp. 459-461, Apr. 15, 2011.

[7] G. J. Foschini, L. J. Greenstein, and G. Vannucci, "Noncoherent detection of coherent lightwave signals corrupted by phase noise," IEEE Trans. Commun., vol. 36, no. 3, pp. 306-314, Mar. 1988.

[8] I. G. Insua, D. Plettemeier, and C. G. Schäffer, "Simple remote heterodyne radio-over-fiber system for gigabit per second wireless access," J. Lightw. Technol., vol. 28, no. 16, pp. 2289-2295, Aug. 15, 2010.

[9] L. Tomba, "On the effect of Wiener phase noise in OFDM systems," IEEE Trans. Commun., vol. 46, no. 5, pp. 580-583, May 1998. 